\title{
A Survey of Vehicular ad Hoc Network Routing Protocols
}

\author{
Jijin Wang*, Xiaoqiang Xiao, Peng Lu \\ College of Computer and Science, National University of Defence and Technology, Changsha, China \\ Email address: \\ Wangiijin13@nudt.edu.cn (Jijin Wang),xqxiao@nudt.edu.cn (Xiaoqiang Xiao), lupeng17@nudt.edu.cn (Peng Lu) \\ ${ }^{*}$ Corresponding author
}

\section{To cite this article:}

Jijin Wang, Xiaoqiang Xiao, Peng Lu. A Survey of Vehicular ad Hoc Network Routing Protocols. Journal of Electrical and Electronic Engineering. Vol. 7, No. 2, 2019, pp. 46-50. doi: 10.11648/j.jeee.20190702.13

Received: April 26, 2019; Accepted: May 20, 2019; Published: May 23, 2019

\begin{abstract}
Vehicular Ad-hoc Network (VANET) is an emerging autonomous dynamic topology network. It is a special kind of Mobile Ad-hoc Network in which the vehicles exchange their information with each other. VANET turns every car in it into a mobile node and use these nodes to create a mobile dynamic network. The purpose of VANET is to supply a wireless connectivity and deploy various applications such as collision avoidance, safety and improving the traffic efficiency as provisioned by the Intelligent Transportation System (ITS). The vehicles are constrained by the realistic traffic environment, and now the simulations are mainly network simulations which cannot simulate the real trace of the vehicle. But as nodes in VANETS have very high mobility, so there are lots of challenges to route the packets to there final destination which need to be addressed by existing/proposing new solutions for the same. Keeping view of above, In this paper, We summarize the existing VANET routing protocols and classify and compare them. Then, We list several classic routing algorithms and analyze their characteristics and advantages and disadvantages. Finally, by analyzing the status quo of vehicle-mounted routing protocols, we illustrate the difficulties and challenges that vehicle-mounted routing protocols will encounter in the future.
\end{abstract}

Keywords: VANET, Routing Protocols, ADOV, OLSR, GSPR, V2V

\section{Introduction}

Vehicular ad hoc Network is a special network architecture composed of vehicle-mounted units and roadside facilities (RSU), connected with cellular network, Wi-Fi and land wireless equipment through wireless data transmission, and then sent to mobile management center through public network. Vehicular Ad-hoc Network is a special category of Mobile Ad-hoc Network (MANET). It has a high-speed and dynamic topology, which is limited by obstacles such as single-path planning and buildings, random density distribution of network nodes, intermittent interruption of wireless transmission and shadow effect. In recent years, the proposal of smart city has brought more attention to VANET [1]. The service provided by VANET has also shifted from the traditional driving safety requirements to more reliable and comprehensive entertainment and living demands. Therefore, the demand for efficient VANET routing protocols is increasingly strong.

The VANET routing is derived from the mobile self-organizing network routing protocol. In order to enable the routing protocol to reach the maximum throughput rate under the condition of minimum packet loss rate and load control, VANET gradually develops various types of routing protocols [2].

\section{Classification of VANET Routing Protocols}

The vehicle-mounted network routing protocol is divided into two categories, (Vehicular to Vehicular) $\mathrm{V} 2 \mathrm{~V}$ and (Vehicular to Infrastructure) V2I respectively. This paper mainly discusses the routing protocol in V2V. There are mainly four types of $\mathrm{V} 2 \mathrm{~V}$ routing protocols: topology based routing protocol, location based routing protocol, cluster based routing association, region multicast routing protocol. The classification of corresponding routes is shown in Figure 1 [2-6].

Topological routing forwarding data through existing links in the network. It includes active routing, passive on-demand routing and mixed routing driven by routing table. On-demand routing can update routing information in a timely manner, but flooding is used to find links, which leads 
to increased routing load and network security problems. Hybrid routing refers to the simultaneous use of two modes to divide nodes into regions according to the network status, and different routing modes within and between regions, so as to improve routing efficiency and reliability [7, 8]. Typical topological routing includes active route Optimized Link State Routing (OLSR), passive route Ad Hoc On Demand Distance Vector (AODV) and hybrid route ZRP. In VANET, due to the high-speed movement of nodes, active routing requires a large amount of bandwidth and a large amount of routing table information. The link found in passive routing is likely to be disconnected soon, so this type of routing is not suitable for vehicle-borne network.

(I) In location-based routing, nodes need to know the location information of themselves and their neighbor nodes, and such routing does not need to maintain routing tables or exchange link status information with neighbor nodes. Location-based routing can also be divided into non-delay tolerant network routing and delay tolerant network routing. The target of the former is to transfer data packets to the target node as soon as possible, which is generally used in effectively populated VANET. The network environment where the latter is located lacks a stable and persistent end-to-end path, and the node will move the data packet before finding the appropriate forwarding node [9] [10]. Greedy perimeter stateless routing (GPSR) protocol is a typical location-based routing, whose basic idea is greedy algorithm, which can enable messages to reach the destination as soon as possible in the intensive network.
However, its performance is not good in the sparse network, and its packet loss rate and transmission delay will greatly increase.

(II) Cluster routing is generally more suitable for networks with clustering topology. Each cluster has a cluster head for intra-cluster and inter-cluster communication, while other nodes can only communicate directly with nodes of the same cluster. In this type of routing, the nodes close to each other form a cluster, and the composition of the cluster and the selection of the cluster head are very important $[11,12]$. Different network types may choose cluster heads in different ways. Its typical routing CBR algorithm has good performance in small networks, and in some areas of urban vehicle-mounted networks, the performance of the algorithm may be poor due to the insufficient number of nodes.

(III) Regional multicast routing is actually a location-based multicast routing that forwards messages to all vehicle nodes in a region. The key is the selection of special geographic region or the definition of Zone Of Relevance (ZOR). Typical routes include IVG and AGR routes [13]. One drawback of such protocols is network partitioning and the presence of harmful neighbor nodes, which can hinder the proper forwarding of messages. Broadcast routing is commonly used in VANET to share information such as road conditions, weather and emergencies. Broadcast routing is message-passing in the form of flooding, where each node forwards the received message to other nodes. This method can guarantee the target to receive information quickly and accurately, but it will cause great load [14].

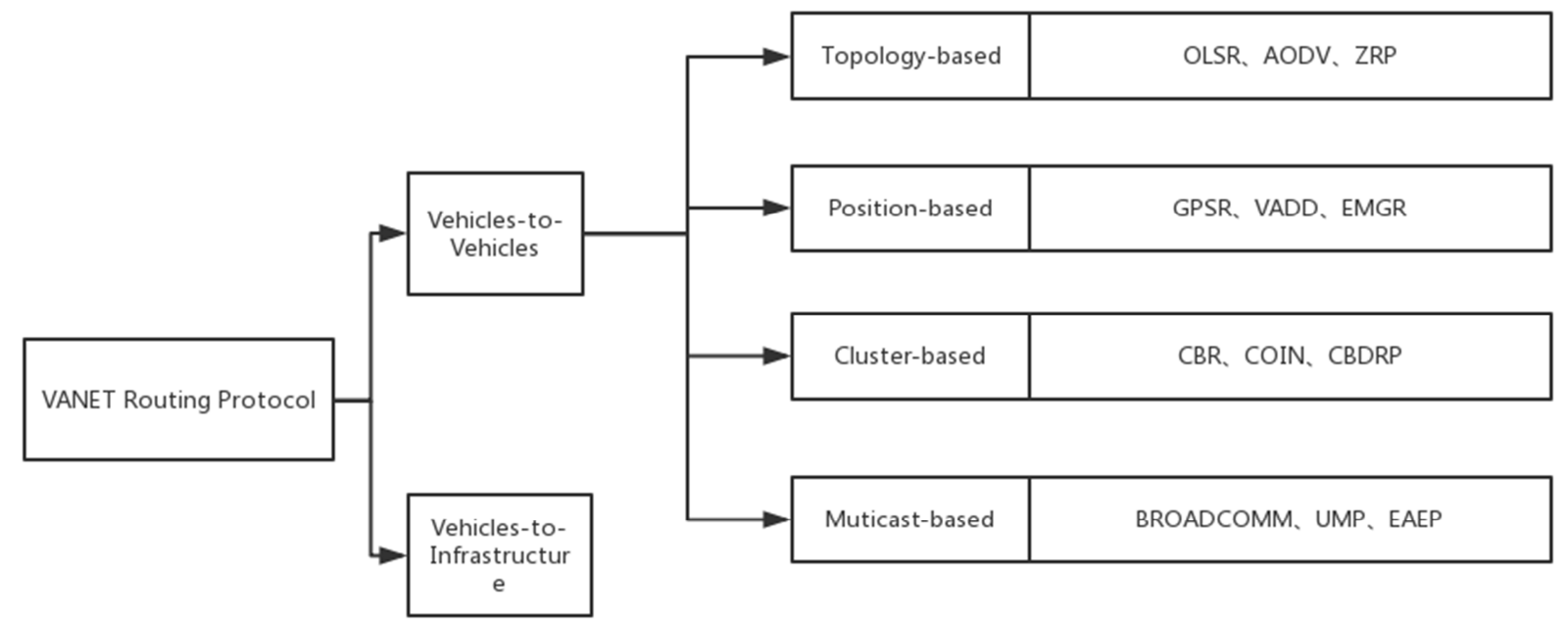

Figure 1. Routing protocol classification.

\section{Classical VANET Routing Protocols}

High dynamic topology characteristics turn out the efficient VANET routing protocols design to be more hard. The VANET routing protocol can be classified into two categories such as Topology based routing protocols and Position based routing protocols, the most popular sub categories under them is Dynamic Source Routing (DSR), Ultra Mobile Broadband (UMB), Optimized Link State Routing(OLSR), Gateway Routing Protocol (GRP) and Ad
Hoc On Demand Distance Vector (AODV) [15].

(1) Topology based routing protocols use links information to transmit the packets of data between

nodes through the VANET. There are two sub categories under this mechanism, the proactive approach which depends on routing techniques related to table driven methodology and the reactive approach which depends on routing techniques related to on demand methodology [15].

(a) Proactive routing protocols are commonly depending on algorithms related to shortest route. They save all the data 
related to the connected nodes in predefined tables which are the main mechanism in these routing protocols. Also, there data are engaged with the partner nodes. Each routing table is updated by its node when the network topology is changed by any event. Advantages: Real time applications low latency; It is not required to have a path discovery. Disadvantages: A huge part of the available bandwidth occupied by unused routes.

(b) Reactive routing protocols are commonly depending on algorithms related to on demand actions. When two nodes want to communicate, they initiate the path discovery and one of its main benefits is the network traffic reduction [16].

Advantages: Flooding is required when it is requested, so it doesn't require proactive overflow in the network; It controls the bandwidth as it is Beaconless.

Disadvantages: Nodes communication disturbance occurred because of the network exaggerated flooding; High latency in path searching.

(2) Geographic based routing protocols are depending on algorithms related to the positioning.

Mechanism using location based applications (For example GPS). Such applications are providing.

Such data for path selection. Also these protocols are not servicing any tables related to routing.

Data or any information related to the join status with the nearby nodes [16].

\section{1. $A O D V$}

Ad-Hoc On Demand Distance Vector routing protocol is depending on a mechanism related to on-demand approach which initiates a path when a VANET node transmits packets of data to another node. The Destination Sequence Number is used by this protocol which is a unique feature not available in similar sub category routing protocols. It can be used in singular and multimode routing [17]

Like all reactive protocols, the philosophy in AODV, the information is only transmitted between nodes in an on-demand mode. When a node wants to transmit traffic to the host node without a predefined route, it will create a (RREQ) route request message to be flooded to the other nodes in a limited way [18], Figure 2 describes the way how AODV works.

AODV uses the below types of control messages for route servicing:

(1) RREQ - When a node is seeking a path to a node, then it transmits the route request message.

(2) RREP - A route reply message is transmitted in a single mode back to the source of a RREQ if the receiver is the node using the required address or it has a functional path to the required address

RERR - In functional paths nodes observes upcoming hops link's status. For reporting technique activation, a "precursor list" is retained by each node, which including its neighbors IP address probably to exercise it as a next hop among each destination node. When the broken link in an active route is detected, other nodes are warned by this message type for the link loss.

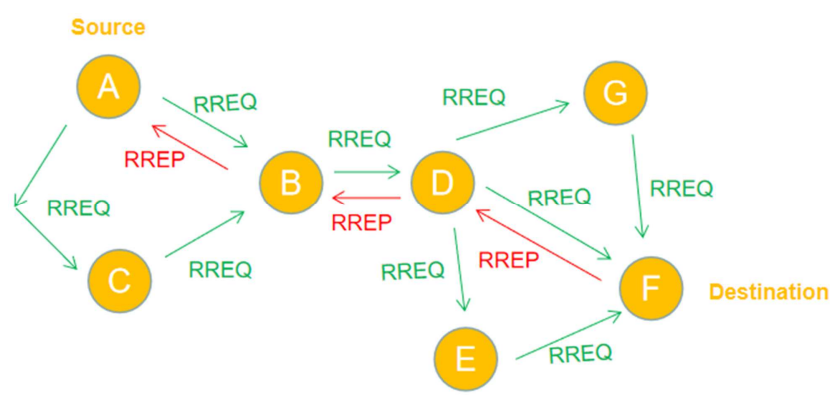

Figure 2. The work principle of $A O D V$.

Advantages: AODV can be used in large VANET networks.; Any failure in the VANET links is handled in a prompt way by the AODV; The route redundancy and excessive memory requirements are minimized.; Distance Sequence Number is providing recent route to the destination node.

Disadvantages: It expends extra bandwidth, because of proactive beaconing High control overhead is occurring when many route reply packets for a single path; Compared to other approaches, high processing time is required for the connection initiation and the first attempt to set the path; Route inconsistency may occurs when old entries are included in intermediate nodes.

\subsection{DSR}

The Dynamic Source Routing Protocol (DSR) utilizes source routing and maintains functional paths. It consists of route detection and route servicing [19].

A node requires four essential structures of data that are considered to be conceptual, to be able to engage in the DSR: a Retransmission Buffer, a Send Buffer, a Route Cache and a Route Request Table [20], the work principle of DSR is display in Figure 3.

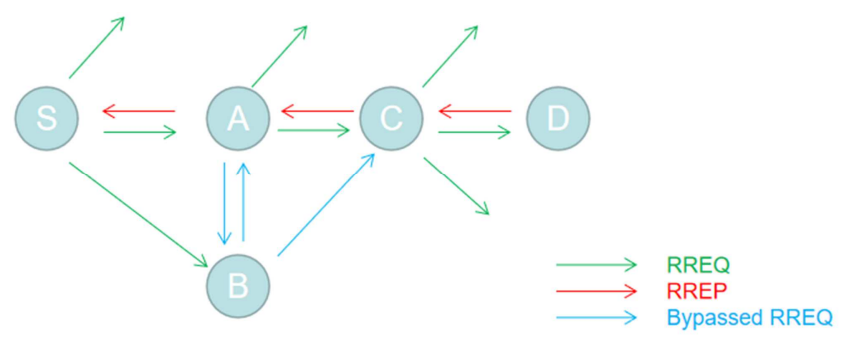

Figure 3. The work principle of DSR

(1) Route Request Table: The route request table is partitioned by the target home address of the route discovery. The Route Request Table is considered of records collection about Route Request packets that were recently forwarded or originated by this node [20].

(2) Route Cache: In the VANET network every node is servicing its own tables which save the route cache. Route Cache is responsible for storing all requested information related to routing by a new participant node in a VANET network using a DSR routing protocol [20].

(3) Retransmission Buffer: The Retransmission Buffer of a node is packets queue sent by this node that is expecting for 
the arrival of an acknowledgment from the next hop in the source path [20].

(4) Send Buffer: Every packet after being registered in the buffer should be deleted from the send buffer and get rid of it in SEND $\backslash$ BUFFER $\backslash$ TIMEOUT seconds, also is associated with the time it is registered into the buffer [20].

Advantages: No proactive updates are desired in DSR; Compared to other approaches, extra overload is occurring on the VANET as it searches for the paths in a reactive approach; Beacon less.

Disadvantages: Cracked links cant be reformed locally; The performance is declining in highly dynamic VANET; The VANET is overflowed by superfluous load; In high traffic VANET network which is an expected pattern, Byte overhead is occurring by the path data in the header.

\subsection{OLSR}

It means optimized link state routing which means a routing protocol using the proactive mode. In this, whenever any change in the topology occur, MPR (multipoint relay) are responsible to generate and forward the topology information to selected nodes [21].

It is a proactive protocol based on the table-driven methodology. from its name, the link-state scheme is used by this protocol in an enhanced way to circulate topology information. OLSR is using this mechanism also, but in order to maintain bandwidth the message overflow in OLSR is enhanced as the protocol works in wireless multi-hop scenarios [21].

AS OLSR(Optimized Link State Routing) protocol based on tables, OLSR operation fundamentally consists of servicing and updating information in a set of tables. These tables are including data which is based on received control traffic, and control traffic is produced based on information returned from these tables. the tables are managing the route calculation itself as well [21].

OLSR uses the below essential control messages types:(1) Topology Control messages (TC); (2) HELLO control messages (HELLO); (3) Multiple Interface Declaration messages (MID).

Advantages: In broadcast scenario, reduce the number of retransmission of packets.

Disadvantages: In OLSR, large amount of bandwidth and CPU power is required to compute the optimal path. GRP routing is used into two approaches. In greedy forwarding, the data is sent to the closest neighbor of the destination node using the three VANET routing mechanisms. In order to select the neighbor node, so these routing mechanisms will be used. the second approach is face- 2 or perimeter routing which implies planner graph traversal concept [17].

In order use the Greedy forwarding approach, the sender node determines the receiver node'sestimated location. The message is transmitted to the receiver node's closest neighbor.

The positioning scheme is responsible for collecting the message for example GPS. The intermediate node receives the data to a neighbor two-faced through the receiver node's way [22]. This process is persistent until the the receiver node receives the data. In the VANET network each node is servicing its own table where the address of the each node is registered [23].

The different routing mechanisms in the greedy forwarding are known as in terms of development, space and direction towards the receiver node [24].

From the different strategies, a node can choose to determines the packet should be transmitted to which neighbor node. The greedy forwarding main obstacle is to choose which neighbor node is the most accurate node to send the data to it. The different routing mechanisms are used For the neighbor node selection.

The three different routing mechanisms in Greedy forwarding approach are Nearest with Forwarded Progress (NFP), Most Forwarded within R (MFR) and Compass Routing. Another approach which is defined as the Perimeter approach or Face-2 method in order to avert the limitations of the greedy forwarding approach. In this routing technique type, if the forward path cannot be determined by any node in the network, so the node with the least backward progress will receive the packet [24].

One of the main drawbacks of this approach that the looping packets problem is existing, which doesn't exist in forward packet towards the destination with positive progress. The Perimeter approach is based on the planner graph traversal, so any node doesn't require to save any extra or unimportant information. by default it progresses to the greedy forwarding mode when the packet becomes more closer to the destination where the packet step inside the improvement mode [23].

Advantages: Route discovery and management is not required; Scalability; Suitable for high node mobility pattern.

Disadvantages: It requires position determining services; GPS device doesn't work in tunnel because satellite signal is absent there.

\section{Conclusion}

This paper presents VANETS by summarizing the existing VANET routing protocols and classify and compare them, and analyzing their characteristics and advantages and disadvantages. Although significant research has already been done, many key factors for their success are still open. There is lack of profound performance evaluation of different schemes and versatile and comprehensive real-life scenarios in VANET context. The few studies that are currently available are not only limited in scope, but also restricted to a specific scenario. Hence, some upcoming challenges are still open to researchers. With the development of VANET and other technologies such as machine learning d data mining, 5G. What we should face is the question to find more effective and available Routing Protocols by using the advanced tools, and We need to highlight the security of the Routing protocols. Maybe we can establish a machine learning model based on the quality of previous routing results to provide basis and reference for later routing. Maybe we can build a more stable transmit road for the VANET 
packets. Routing Protocols is the most important part of VANET which determined the smart city's direction, it also will meet challenges among security, we also have a long way in this field. The solution should consider the least hops and the most stable way.

\section{References}

[1] Bonola M, Bracciale L, Loreti P, et al. Opportunistic communication in smart city: Experimental insight with small-scale taxi fleets as data carriers [J]. Ad Hoc Networks, 2016, 43: 43-55.

[2] Nahrstedt K. 3R: Fine-grained encounter-based routing in Delay Tolerant Networks [C] // IEEE International Symposium on A World of Wireless, Mobile and Multimedia Networks. IEEE Computer Society, 2011: 1-6.

[3] Vegni A M, Loscrí V. A Survey on Vehicular Social Networks [J]. IEEE Communications Surveys \& Tutorials, 2015, 17 (4): 2397-2419.

[4] Patel D, Faisal M, Batavia P, et al. Overview of Routing Protocols in VANET [J]. International Journal of Computer Applications, 2016, 136.

[5] Sharef B T, Alsaqour R A, Ismail M. Vehicular communication ad hoc routing protocols: A survey [J]. Journal of Network \& Computer Applications, 2014, 40 (1): 363-396.

[6] Ding Y, Qu H, Wang X. An improved AODV routing protocol for high moving VANET [C] // Electronic System-Integration Technology Conference. 2012: 100-103.

[7] AK Singh, RS Rao, AS Baghel. Position Based Routing Protocols for Vehicular Ad Hoc Network: A Review [J]. IJCSMC, Vol. 5, Issue. 2, February 2016, pg.206 - 212.

[8] Karp B, Kung H T. GPSR: greedy perimeter stateless routing for wireless networks [C] // International Conference on Mobile Computing and NETWORKING. ACM, 2000: 243-254.

[9] Chen Y, Weng S, Guo W, et al. A Game Theory Algorithm for Intra-Cluster Data Aggregation in a Vehicular Ad Hoc Network [J]. Sensors, 2016, 16 (2): 245.

[10] Satyajeet D, R. A, S. S. Heterogeneous Approaches for Cluster based Routing Protocol in Vehicular Ad Hoc Network (VANET) [J]. International Journal of Computer Applications, 2016, 134.
[11] Sharef B T, Alsaqour R A, Ismail M. Vehicular communication ad hoc routing protocols: A survey [J]. Journal of Network \& Computer Applications, 2014, 40 (1): 363-396.

[12] VA Gajbhiye, RW Jasutkar. Study of Efficient Routing Protocols for VANET. [J]. International Journal of Scientific \& Engineering Research Volume 4, Issue3, March-2013, 1-8.

[13] Paul, B., Faisal, M., \& Naser, A. (2011, April). VANET routing protocols: Pros and cons. arXiv preprint arXiv: 1204.1201

[14] Kaur, H., Singh, H., \& Sharma, A. (2016, February). Geographicrouting protocol: A review. International Journal of Grid and Distributed Computing, 9, 245-254.

[15] Perkins, C., Belding-Royer, E., \& Das, S. (2003, July). Ad hoc on-demand distance vector (AODV) routing.

[16] Hafslund, A. (2003, July). Implementing and extending the optimized link state routing protocol.

[17] Johnson, D. B., \& Maltz, D. A. (1996). Dynamic source routing in ad hoc wireless networks. Mobile Computing, 153-181.

[18] Josh Broch, D. B. J., \& Maltz, D. A. (1998, December). The dynamic source routing protocol for mobile ad hoc networks.

[19] Jacquet, P., Muhlethaler, P., Clausen, T., Laouiti, A., Qayyum, A., \& Viennot, L. (2003). Optimized link state routing protocol (OLSR).

[20] Mauve, M., Widmer, J., \& Hartenstein, H. (2001, December). A survey on position-based routing in mobile ad ho networks. IEEE Network, 15, 30-39.

[21] Sharma, V., Kaur, M., \& Singh, H. (2012). A survey on reactive ad hoc routing protocols in MANET. CiiT International Journal of Wireless Communication.

[22] Maghsoudlou, A., Sthilaire, M., \& Kunz, T. (2011, October). A survey on geographic routing protocols for mobile ad hoc networks.

[23] Khatkar, A., \& Singh, Y. (2012, October). Performance evaluation of hybrid routing protocols in mobile ad hoc networks. In Advanced Computing \& Communication Technologies (ACCT), 2012 Second International Conference (pp. 542-545). IEEE.

[24] Menon, V. G., \& Prathap, J. P. (2013, August). Performance analysis of geographic routing protocols in highly mobile Ad hoc network. Journal of Theoretical \& Applied Information Technology, 54. 